\title{
Structure and Collapse of a Surface-Grown Strong Polyelectrolyte Brush on Sapphire
}

Iain E. Dunlop, ${ }^{* \dagger, \ddagger}$ Robert K. Thomas, ${ }^{\dagger}$ Simon Titmus, ${ }^{\dagger, \S}$ Victoria Osborne,, Steve Edmondson, ${ }^{\|, \perp}$ Wilhelm T.S. Huck, ${ }^{\|, \nabla}$ and Jacob Klein ${ }^{*}, \dagger, \#$

${ }^{\dagger}$ Department of Chemistry, Physical and Theoretical Chemistry Laboratory, University of Oxford, South Parks Road, Oxford, OX1 3QZ, U.K.

"Melville Laboratory for Polymer Synthesis, University Chemistry Laboratory, Lensfield Road, Cambridge, CB2 1EW, U.K.

\#Department of Materials and Interfaces, Weizmann Institute of Science, P. O. Box 26, Rehovot 76100, Israel

\section{Supporting Information}

ABSTRACT: We have used neutron reflectometry to investigate the behavior of a strong polyelectrolyte brush on a sapphire substrate, grown by atom-transfer radical polymerization (ATRP) from a silane-anchored initiator layer. The initiator layer was deposited from vapor, following treatment of the substrate with an $\mathrm{Ar} / \mathrm{H}_{2} \mathrm{O}$ plasma to improve surface reactivity. The deposition process was characterized using $\mathrm{X}$-ray reflectometry, indicating the formation of a complete, cross-linked layer. The brush was

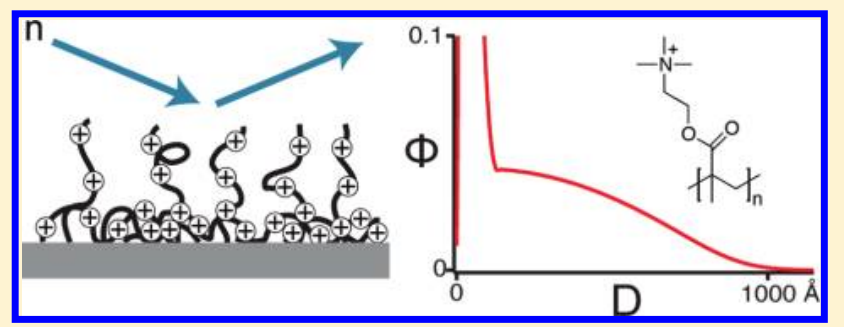
grown from the monomer [2-(methacryloyloxy)ethyl] trimethyl-

ammonium chloride (METAC), which carries a strong positive charge. The neutron reflectivity profile of the swollen brush in pure water $\left(\mathrm{D}_{2} \mathrm{O}\right)$ showed that it adopted a two-region structure, consisting of a dense surface region $\sim 100 \AA$ thick, in combination with a diffuse brush region extending to around $1000 \AA$ from the surface. The existence of the diffuse brush region may be attributed to electrostatic repulsion from the positively charged surface region, while the surface region itself most probably forms due to polyelectrolyte adsorption to the hydrophobic initiator layer. The importance of electrostatic interactions in maintaining the brush region is confirmed by measurements at high $(1 \mathrm{M})$ added 1:1 electrolyte, which show a substantial transfer of polymer from the brush to the surface region, together with a strong reduction in brush height. On addition of $10^{-4} \mathrm{M}$ oppositely charged surfactant (sodium dodecyl sulfate), the brush undergoes a dramatic collapse, forming a single dense layer about $200 \AA$ in thickness, which may be attributed to the neutralization of the monomers by adsorbed dodecyl sulfate ions in combination with hydrophobic interactions between these dodecyl chains. Subsequent increases in surfactant concentration result in slow increases in brush height, which may be caused by stiffening of the polyelectrolyte chains due to further dodecyl sulfate adsorption.

\section{INTRODUCTION}

Polyelectrolyte brushes are of great interest as systems that are simple enough to be understood theoretically but are important in many situations of practical importance. For example, polyelectrolyte brushlike structures are important for the stabilization of colloidal casein particles in milk $^{1}$ and have been implicated in lubrication at the ocular surface, ${ }^{2-5}$ while synthetic polyelectrolyte brushes have found applications including the stabilization of colloidal suspensions of synthetic nanoparticles ${ }^{6}$ and production of responsive, protein resistant, and biofunctional surfaces. $^{7-9}$ The properties of polyelectrolyte brushes, including their structure, ${ }^{10-12}$ mechanical and lubrication properties, ${ }^{13-16}$ and their responses to changing $\mathrm{pH}$ and electrolyte conditions, ${ }^{10,11,16-23}$ have thus been widely investigated (for reviews see, e.g., refs 24-27).

Synthetic polyelectrolyte brushes can be prepared by two distinct approaches: either via the self-assembly of preexisting polymer molecules ("grafting-to") or by first decorating the substrate surface with functional groups that act as polymerization initiators from which polymer chains can be grown in situ ("grafting-from"). Recent years have seen increasing interest in the grafted-from approach, due to the development of modern synthetic approaches based on controlled/living radical polymerizations, such as atom-transfer radical polymerization (ATRP). Notably, acrylate- and methacrylate-based ATRPs have been used to create a wide variety of brushes with different functionalities and architectures, based on a common synthetic approach (for reviews, see, e.g., refs 28-30). The advantages of grafted-from brushes include the ability to access higher density regimes, due to bypassing the kinetic limitations on grafted-to brush density that arises from the slow diffusion of preexisting polymer chains through a partially formed brush. ${ }^{18,31-33}$ Also, it is generally straightforward to devise brushes that are strongly surfaceanchored, for example through the use of covalently anchored

Received: November 25, 2011

Revised: January 6, 2012

Published: January 31, 2012 
self-assembled monolayers of initiator molecules. Finally, the use of grafting-from can bypass physical limitations that apply to the self-assembly of preexisting polymer, e.g., by permitting unlimited overcompensation of the electrical charge on a substrate surface. $^{34}$

Specular neutron reflection, which is the principal technique used in this study, has proved especially valuable in investigating polyelectrolyte brushes, enabling in situ determination of static structures at the solid-liquid interface, on length scales in the range of 1-2000 $\AA$. This technique has been applied to brushes prepared using both grafting-to ${ }^{8,10,35-37}$ and graftingfrom methods. Recently, a number of studies have been performed of grafted-from brushes prepared using methacrylate ATRP, focusing on a variety of weak $\mathrm{pH}$-responsive brushes, ${ }^{11,21,34,38,39}$ as well as strong polyelectrolyte brushes ${ }^{21,40,41}$ and polyampholytes. ${ }^{22}$

In this study, we investigate a strong polyelectrolyte brush, determining its response to both added electrolyte and oppositely charged surfactant. In contrast to most previous neutron reflectometry studies, which have investigated brushes on silicon or quartz substrates, we use a sapphire single crystal whose scattering length density (effectively the neutron refractive index) is close to that of $\mathrm{D}_{2} \mathrm{O}$. This gives an enhanced signal since, in the case of a nondeuterated brush, a greater proportion of the reflected intensity derives from the brush itself rather than from the contrast between substrate and solvent. For this reason, sapphire substrates offer substantial promise for neutron reflectometry investigations of low-density structures such as polymer and polyelectrolyte brushes, especially in systems that incorporate components that are difficult to deuterate, such as biological molecules.

A feature of the present study is that we use a trimethoxysilanebased self-assembled monolayer as the basis for initiator functionalization of the sapphire substrate, whereas the small number of previous studies using this substrate have relied on electrostatic immobilization. ${ }^{34,39}$ The trimethoxysilane approach has the advantage of strong anchoring to the substrate, based on a covalently bonded cross-linked layer. This approach is generalizable well beyond polymer brushes, due to the ready availability of trimethoxysilane molecules with a wide range of functionalities. The present study thus serves as a proof-of-principle for this method.

\section{EXPERIMENTAL SECTION}

Synthesis of Polyelectrolyte Brushes. Preparing an InitiatorFunctionalized Surface. A sapphire single-crystal substrate whose front surface $(126 \mathrm{~mm} \times 50 \mathrm{~mm})$ had been made flat by lapping and polishing was incubated in a fresh mixture of sulfuric acid, hydrogen peroxide, and ultrapure water (volume ratio, $4: 1: 5$ ) at $80^{\circ} \mathrm{C}$ for $15-20$ min to remove organic contamination, then rinsed with ultrapure water, blown dry with filtered nitrogen, and treated for $2 \mathrm{~min}$ at $6.8 \mathrm{~W}$ radio-frequency power in an argon-water plasma (Basic Plasma Cleaner, Harrick Scientific Corp., Ossining, NY, USA), to ensure the presence of pendant -OH groups. The crystal was incubated in a mixed vapor of 3-(trimethoxysilyl)propyl 2-bromo-2-methylpropionate, which we will call silane initiator (Figure 1b), and hexane for $65 \mathrm{~h}$. (A beaker containing $10 \mathrm{~mL}$ of hexane (anhydrous, Sigma-Aldrich, St. Louis, MO, USA) and silane initiator ( $0.25 \mathrm{~g}$, custom synthesized by Gelest, Inc., Morrisville, PA, USA), stored anhydrously, was placed in a $2 \mathrm{~L}$ desiccator along with the crystal; the desiccator was exposed to vacuum (0.6 L PTFE diaphragm pump, Leybold Vacuum, Cologne, Germany; minimum pressure $\sim 8 \mathrm{mbar}$ ) and then sealed to permit the crystal surface to react with the vapor.) The sapphire surface was thus functionalized with $-\mathrm{O}-(\mathrm{C}=\mathrm{O})-\mathrm{C}\left(\mathrm{CH}_{3}\right)_{2} \mathrm{Br}$ groups that can act as initiators for acrylate ATRP.

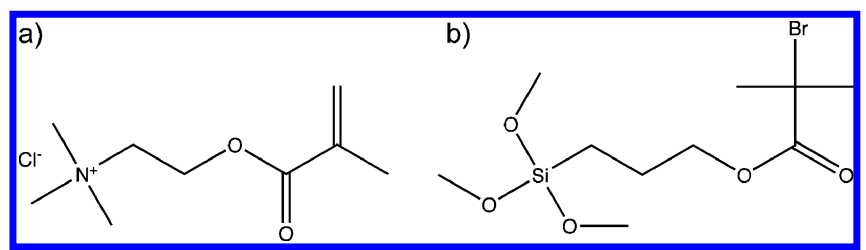

Figure 1. (a) [2-(Methacryloyloxy)ethyl]trimethylammonium chloride (METAC); (b) 3-trimethoxysilylpropyl-2-bromo-2-methylpropionate (silane initiator).

Atom-Transfer Radical Polymerization. The polyelectrolyte brushes were synthesized from the monomer [2-(methacryloyloxy)ethyl]trimethylammonium chloride (METAC; Figure 1a). The electrical charge of the brush in aqueous conditions arises from the quaternary ammonium group on each METAC monomer. The synthesis protocol has been published previously. ${ }^{16}$ All steps were carried out under ambient conditions. METAC solution (100 g of 75 wt \% water, SigmaAldrich) was mixed with ultrapure water $(100 \mathrm{~mL})$. The solution was adjusted to $\mathrm{pH} 8$ with aqueous sodium hydroxide. $N, N^{\prime}$-Bis[2-(dimethylamino)ethyl]- $N, N^{\prime}$-dimethylethane-1,2-diamine (HMTETA; $2.2 \mathrm{~mL}$, Sigma-Aldrich), copper(I) chloride (anhydrous, $0.143 \mathrm{~g}$ ), and copper(II) chloride (anhydrous, $0.88 \mathrm{~g}$ ) were added, and the solution was stirred for $15 \mathrm{~min}$ to ensure dissolution. The sapphire crystal was incubated in the resulting solution for $15 \mathrm{~min}$ to allow the polymerization to occur and was then removed, rinsed with ultrapure water and blown dry with filtered nitrogen. The crystal surface was examined by X-ray reflectometry to confirm that a brush had been successfully grown. Neutron reflectometry measurements were begun within $24 \mathrm{~h}$ : in the meantime the crystal was stored under ambient conditions.

In preliminary experiments to characterize and optimize the synthesis protocol, poly(METAC) brushes were grown on commercially polished sapphire wafer pieces $(40 \mathrm{~mm}$ (axis parallel to X-ray beam) $\times 20 \mathrm{~mm}$ ) using a procedure identical to that described in preceding text except that the wafers were not acid-cleaned, and the incubation time in the silane initiator-hexane vapor was varied.

Specular Neutron Reflectometry. For an introduction to the principles of specular neutron reflectometry, see, e.g., ref 42 . Briefly, a neutron beam passes through a solid substrate, in this case a sapphire crystal, and is incident on the solid-liquid interface. Total reflection occurs if the angle between the incident beam and the interface, $\theta$, is less than the critical angle $\theta_{\mathrm{c}}$ given by $\sin \theta_{\mathrm{c}}=\lambda\left(\delta_{\rho} / \pi\right)^{1 / 2}$, where $\lambda$ is the de Broglie wavelength of the neutrons and $\delta_{\rho}$ is the difference between the scattering length density of the solid and the liquid subphase; the scattering length density, $\rho$, is defined to be the product of the number density of atoms with the scattering length of a neutron from a single atom. Above the critical angle, the reflectivity, $R(\theta)$, falls off rapidly with increasing $\theta$. In practice, $R$ is usually given as a function of the momentum transfer, $q=(4 \pi / \lambda) \sin \theta$, where $\lambda$ is the de Broglie wavelength of the neutrons. Superimposed on this falloff is a function $g(q)$ that gives information on the structure of the interface, that is, on the neutron scattering length density of the liquid phase as a function of distance from the solid surface, $f(z)$. For $q \gg q_{c}=(4 \pi / \lambda) \sin$ $\theta_{\mathcal{c}}$ when the so-called kinematic approximation applies, $g(q)$ is proportional to the Fourier transform of $f(z)$; more generally, it may be taken that features in $g(q)$ at high $q$ correspond to short length scale features in $f(z)$ and vice versa. For structures consisting of multiple layers of varying scattering length density, calculations of the reflectivity valid for all $q$ can be made using the so-called optical matrix method or Parratt algorithm. ${ }^{43}$ In this study we follow the common practice of using a multilayer model for the studied structures and adjusting the model parameters until the reflectivity calculated using the optical matrix method corresponds to the measured reflectivity.

Specular neutron reflectometry measurements were made using the SURF reflectometer at the ISIS neutron source, Rutherford Appleton Laboratory, Chilton, U.K. ${ }^{44}$ Solution changes were made by thorough rinsing with water and/or $\mathrm{D}_{2} \mathrm{O}$, followed by injection of the desired solution immediately before measurement. The sample holder was 
dismounted and remounted on a kinematic mount between measurements, introducing errors in the incident angle, $\theta$, which were allowed for by adjusting data so that the critical angle for total internal reflection was consistent (allowing for scattering length density variations between the different subphase solutions). Neutron scattering length densities were taken from the literature (sodium dodecy sulfate (SDS), $\left.0.398 \times 10^{-6} \AA^{-2} ; \mathrm{D}_{2} \mathrm{O}, 6.35 \times 10^{-6} \AA^{-2}\right)^{45}$ or calculated from tabulated chemical formulas and densities (sapphire, $5.72 \times 10^{-6} \AA^{-2}$; METAC, $0.77 \times 10^{-6} \AA^{-2}$; silane initiator, $\sim 0.4 \times 10^{-6}$ $\AA^{-2}$ ). Since the scattering length densities of the organic species are very similar in comparison with that of $\mathrm{D}_{2} \mathrm{O}$, they may be taken as identical to a good approximation, following an approach used previously. ${ }^{39}$ Thus, fitting was used to determine the total surface excess of organic material, $\Gamma$, assuming that all material present has the same scattering length density as poly(METAC); surface excesses of individual species, $\Gamma_{i}$, were then determined from $\Gamma=\sum_{i} \Gamma_{i}$.

Modeling and Fitting Data. Neutron reflectivity profiles for the surface-grown polyelectrolyte layer in pure water and electrolyte solutions were fitted with a model that divided the polyelectrolyte layer into two regions: a dense surface region of polyelectrolyte in the vicinity of the surface, with a more diffuse brush region above this. To allow the use of the optical matrix method to calculate the reflectivity profile, this picture was implemented as a multilayer mode. A precise description of the model follows.

Surface Region. This region was divided into two layers, as the minimum required to obtain a good fit, and was thus defined by the thickness $\left(d_{1}, d_{2}\right)$, polymer volume fraction $\left(\phi_{1}, \phi_{2}\right)$ and roughness $\left(\sigma_{1}, \sigma_{2}\right)$ of each of these layers. All of these parameters were varied independently. (The roughness of layer 0 (the sapphire substrate) was determined from X-ray reflectometry measurements.)

Brush Region. This was defined by the total amount of polymer in this region, $\Gamma_{\mathrm{b}}$, the brush height, $h$, and two parameters associated with the shape of the volume fraction profile, which we call brush roughness, $\sigma_{\mathrm{b}}$, and brush roughness increase, $I_{\sigma}$. The brush region was divided into 10 layers for fitting purposes, with the thickness of each layer equal to $h / 10$. The volume fraction of layer $n, \phi_{n}$, is given by

$$
\phi_{n}=\frac{3 \Gamma_{\mathrm{b}}}{2 h^{3}}\left(h^{2}-x_{n}{ }^{2}\right)
$$

where $x_{n}$ is the distance between the center of layer $n$ and that of the outer layer of the surface region, and the outer roughness of layer $n$ (defined such that the substrate is layer zero, so that $n=3$ for the innermost brush layer) is

$$
\sigma_{n}=\left(1+(n-3) I_{\sigma}\right) \sigma_{\mathrm{b}}
$$

where $0 \leq I_{\sigma} \leq 1$, so that if $I_{\sigma}=0$, each of the brush region layers has the same roughness, and the brush layer volume fraction profile is approximately parabolic, but with an error function falloff at the outer edge. As $I_{\sigma}$ is increased toward 1 , the volume fraction profile falls off more slowly with distance from the surface. The parameter $I_{\sigma}$ was introduced to allow for the observed variation of volume fraction profile shape with electrolyte concentration.

Specular X-ray Reflectometry. Characterization of dry brushes in ambient air by specular X-ray reflectometry was carried out using a Bruker D8 Advance X-ray reflectometer (Bruker-AXS, Karlsruhe, Germany), with a home-built sample stage, using $\mathrm{Cu} \mathrm{K} \alpha$ X-rays (wavelength, $\lambda=1.51 \AA$ ). Data analysis was performed using the optical matrix method ${ }^{43}$ as implemented in the Parratt32 program. ${ }^{46}$ In the case of the preliminary experiments on sapphire wafer pieces, a correction to the intensity was made at low incident angles to allow for the effect of the finite sample size.

\section{RESULTS AND DISCUSSION}

Sapphire-Silane Chemistry and Brush Growth. In experiments to determine the effect of the reaction time between the sapphire surface and the silane initiator vapor, poly(METAC) brushes grown as described in the Experimental Section, but with various sapphire-silane initiator vapor reaction times, were characterized by $\mathrm{X}$-ray reflectometry in ambient air after they had been blown dry with dry nitrogen. Xray reflectivity profiles showed prominent interference fringes and could be fitted by treating the brush as a single layer with Gaussian roughness to determine the thickness, scattering length density, and roughness of the film. Figure 2 shows the

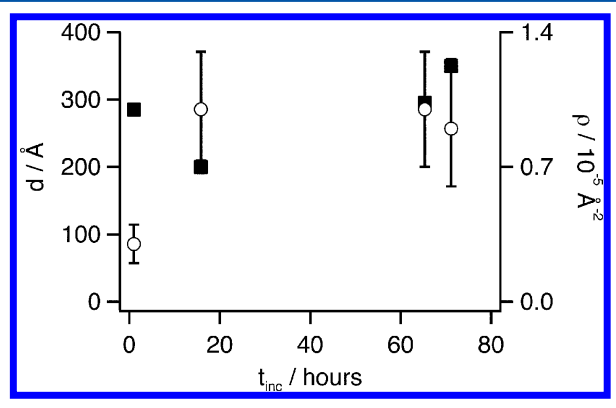

Figure 2. Effect of the sapphire-silane initiator vapor reaction time on the thickness in air, $d$ ( $\mathbf{\square}$, left-hand axis), and scattering length density, $\rho(\mathrm{O}$, right-hand axis), of the eventual "blown-dry" poly(METAC) layer. Values are derived by fitting measured X-ray reflectivity profiles of samples prepared on sapphire wafer substrates, as described in the text. Polymerization conditions were the same for all samples. Error bars show the uncertainty in the fitting (uncertainty in values of $d$ is less than the symbol size). Note that neutron experiments show that this blown-dry layer has a high remaining volume fraction of water.

effect of the sapphire-silane initiator vapor reaction time on the thickness and scattering length density of the eventual brush. It can be seen that the brush thickness had already reached a plateau within the scatter by $1 \mathrm{~h}$ reaction time, whereas the scattering length density reached a plateau only at longer times. These results can be understood if the silane initiator layer grows in islands, where the local silane initiator density within the islands is always saturated and is therefore a constant, regardless of the fraction of the surface covered by the islands. The poly(METAC) brush thickness is determined by this local initiator density and thus also remains constant. The scattering length density, however, depends on the overall surface coverage and thus saturates only slowly with time. This suggested layer growth mechanism based on islands is plausible if cross-linking between adjacent molecules is important for the formation of the layer, as has previously been suggested for alkylsilane layers on sapphire. ${ }^{47}$ The thickness of the initiator layer at itself was shown by X-ray reflectometry of a bare initiator surface to be $10 \AA$, consistent with previously published measurements that used a similar functionalization protocol on plasma-activated mica. ${ }^{16}$ This is thicker than would be expected for a genuine monolayer, suggesting that silane polymerization plays a role in the layer formation. It should be noted that these X-ray measurements do not allow us to distinguish between METAC and water, as these have rather similar X-ray scattering length densities. However, comparing the X-ray "blown-dry" thickness with the values for total polymer coverage obtained from neutron reflectometry measurements below shows that the blown-dry brush is $19 \%$ polymer and $81 \%$ water.

Poly(METAC) Brush: Dry Characterization. The poly(METAC) brush used for the neutron reflectivity experiments was grown from a large home-polished sapphire block and blown dry immediately after synthesis. The X-ray reflectivity of this brush was measured, to confirm that the brush grown was similar to that obtained using commercially available polished sapphire wafers (previous section). A single layer fit to the Xray reflectivity profile gave a "dry" thickness of $215 \AA$, similar to 
those of the wafer-grown brushes, and a scattering length density of $(1.0 \pm 0.1) \times 10^{-5} \AA^{-2}$, identical to that expected for a METAC/water layer that entirely covers the substrate surface.

Poly(METAC) Brush in Pure Water $\left(D_{2} O\right)$ : A TwoRegion Structure. Figure 3a shows the neutron reflectivity

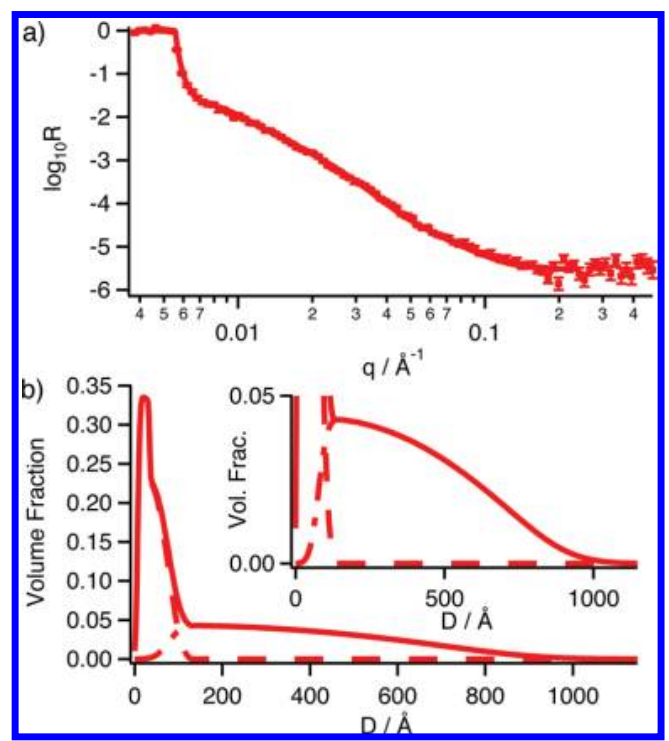

Figure 3. Two-region structure of poly(METAC) brush in pure water $\left(\mathrm{D}_{2} \mathrm{O}\right)$. (a) Neutron reflectivity, $R$, as a function of momentum transfer, q. Symbols show measured data; solid line shows fit to tworegion model (for parameter values see Supporting Information). (b) Polymer volume fraction of profile corresponding to solid-line fit in a. Solid line (-) shows total volume fraction; dashed line (--) shows polymer associated with surface region, and dashed-dotted line (-.-) shows the brush region.

from the sapphire-poly(METAC) brush-water $\left(-\mathrm{D}_{2} \mathrm{O}\right)$ interface. Fitting the reflectivity profile at higher $q$ values required the presence of a thin, dense, surface region, $80 \AA$ thick, and containing about half of the observed material. Although the fitting did not distinguish between silane initiator and the poly(METAC) brush, due to their similar scattering length densities, the thickness of the surface region greatly exceeds that of the $10 \AA$ initiator layer. A further feature of the reflectivity profile is the point of inflection at lower $q$ (around $\left.q=0.08 \AA^{-1}\right)$, indicating that the total swollen thickness of the poly(METAC) brush is large. Upon fitting, it turns out that the remaining half of the poly(METAC) had swollen well away from the surface, forming a diffuse brushlike region which extended to a maximum distance of around $830 \AA$ from the surface. The poly(METAC) brush could thus be seen to have adopted a two-region structure, with some polymer in a thin, dense surface region, and the rest in a brush region that stretches far away from the surface. The overall polymer volume fraction profile obtained by fitting is shown in Figure $3 \mathrm{~b}$.

The polymer volume fraction profile in the brush region was modeled using a stretched parabolic function. Initially, a model that approximated to a parabola $\phi(x) \propto\left(h^{2}-x^{2}\right)$, where $\phi(x)$ is the polymer volume fraction, $x$ is the distance from the surface, and $h$ is the brush height, was tried, since this density profile is theoretically predicted for a (monodisperse) polymer brush. ${ }^{48}$ Such a density profile was unable to precisely reproduce the point of inflection at low $q$, even when a Gaussian falloff at the outside edge was added. A good fit was produced by a density profile with a less sharp falloff. For details of this final model, which was used throughout this paper except where otherwise stated, and the precise meaning of parameters, see the Experimental Section.

The presence of the dense surface region can be rationalized by the adsorption of poly(METAC) chains to the silane initiator-functionalized substrate surface. Since the backbone of the poly(METAC) is essentially hydrophobic, it makes sense for it to exhibit adhesion to the hydrophobic initiator substrate. The amount of polymer in the surface region may also be increased by the presence of some shorter chains due to polydispersity within the brush. Provided there is sufficient polymer in the surface region to give the surface a strong effective positive charge, the surface will be repulsive to the remaining poly(METAC) chains. These remaining chains will then stretch away from the surface due to the osmotic pressure of their confined counterions, forming a classical polyelectrolyte brushlike region with a much lower polymer volume fraction than the surface layer. Such an effect has been proposed theoretically by Zhulina et al., ${ }^{49}$ for a polyelectrolyte brush on an oppositely charged surface, although in the present system, the attraction of the polymer to the surface is likely to be hydrophobic rather than electrostatic. It is interesting to note that this effect would be difficult to observe using a brush formed by grafting-to, since the electrostatic repulsion between polyelectrolyte chains would make it difficult to achieve a sufficiently high surface coverage. A similar two-region structure has recently been reported in a related system. ${ }^{34}$ The idea that the brush region consists of polyelectrolyte that is electrostatically excluded from the surface region is supported by the observation that the amount of polyelectrolyte in the brush is reduced at high electrolyte concentrations (next section).

Effect of Added 1:1 Electrolyte. Figure 4 shows the neutron reflectivity profiles of the poly(METAC) brush in

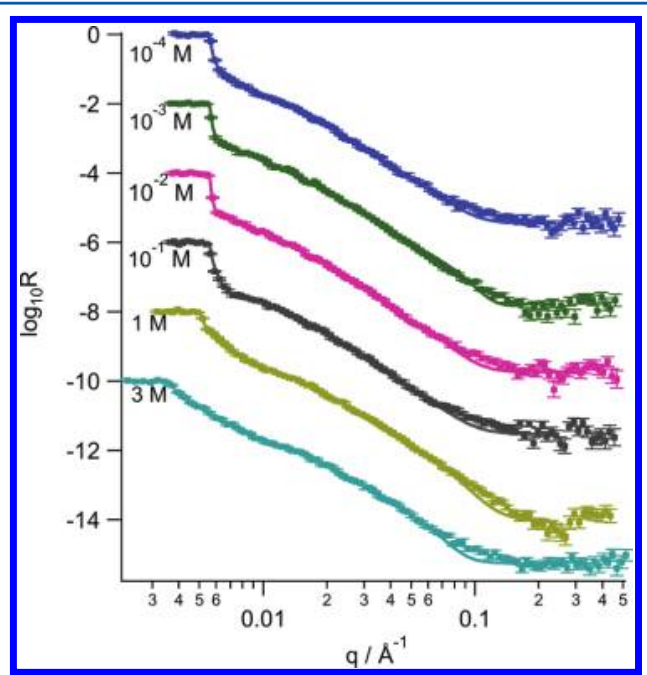

Figure 4. Neutron reflectivity from poly(METAC) brush in increasing concentrations of $\mathrm{KNO}_{3}$ in $\mathrm{D}_{2} \mathrm{O}$. Symbols show measured data; solid lines show fits to two-region model (for parameter values, see the Supporting Information). Successive profiles are shown displaced vertically for clarity. The shifted critical edges at 1 and $3 \mathrm{M}$ are due to the changed refractive index of the aqueous subphase.

solutions of increasing concentration of potassium nitrate in $\mathrm{D}_{2} \mathrm{O}$. All of these data could be fitted with the same two-region model as was used for the brush in pure water: the fitted volume fraction profiles are shown in Figure 5 (see the Supporting Information for full values of fitting parameters). 


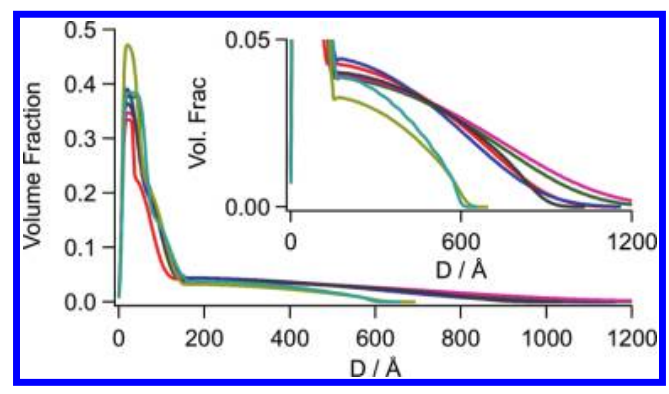

Figure 5. Effect of electrolyte on poly(METAC) layer structure. Polymer volume fraction profile of the poly(METAC) brush in increasing concentrations of $\mathrm{KNO}_{3}$ in $\mathrm{D}_{2} \mathrm{O}$. Lines show the total polymer volume fraction profiles derived from solid-line fits in Figure 4, with different $\mathrm{KNO}_{3}$ concentrations represented by the same colors as in Figure 4. Inset shows a close-up on the brush region (same data as the main figure).

The amount of poly(METAC) in the brush region, $\Gamma_{b}$, remains constant within the scatter at concentrations up to and including $10^{-1} \mathrm{M}$ but roughly halves when the concentration is raised to $1 \mathrm{M}$, as shown in Figure 6a. This effect is likely to arise

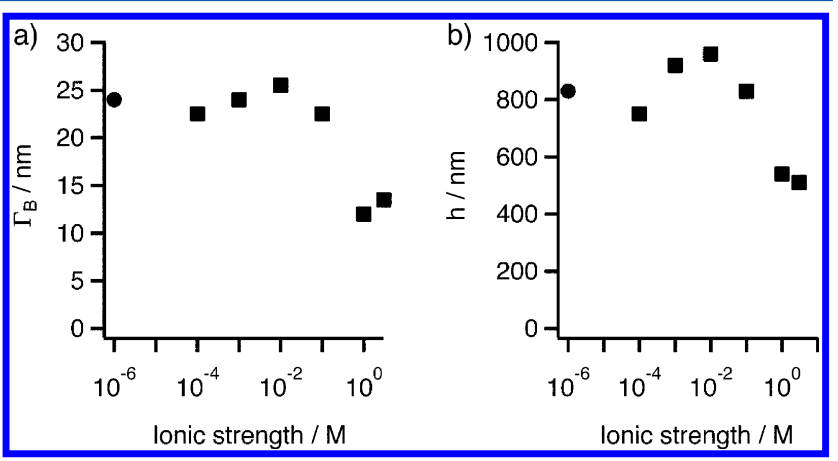

Figure 6. Effect of $\mathrm{KNO}_{3}$ concentration on (a) the volume of poly(METAC) per unit substrate surface area in the brush region, $\Gamma_{\mathrm{B}}$, and (b) the brush height, $h\left(\boldsymbol{\square}, \mathrm{KNO}_{3}\right.$ in $\mathrm{D}_{2} \mathrm{O}$; $\boldsymbol{\bullet}$, pure $\left.\mathrm{D}_{2} \mathrm{O}\right)$.

from the screening out of electrostatic repulsions between the charged chains due to the added electrolyte which enables more chains to join the surface layer. (The total amount of bound organic material remains constant at $48 \pm 4 \AA$ equivalent dry thickness.)

Similarly to $\Gamma_{\mathrm{b}}, h$ (i.e., the thickness of the brush region) remains constant within the scatter at concentrations up to $10^{-1}$ $\mathrm{M}$ but falls dramatically when the concentration is raised to $1 \mathrm{M}$ (Figure 6b; see also the inset to Figure 5). This drop is likely to be due to the reduced number of chains in the brush at $\left[\mathrm{KNO}_{3}\right]=1 \mathrm{M}$ (as evidenced by the fall in $\Gamma_{\mathrm{b}}$ ), in combination with a reduction in the electrostatic repulsion between the chains due to screening by the added electrolyte.

These observed changes in the brush region at high electrolyte concentrations can be considered quantitatively with reference to the work of Pincus, ${ }^{50}$ who showed that a nonadsorbing polyelectrolyte brush should enter the so-called salted brush regime where electrostatic screening becomes important when electrolyte and monomer number densities are of the same order of magnitude (assuming full dissociation of counterions from the polyelectrolyte). This is because the osmotic pressure of added electrolyte is then equal to that of the brush-confined counterions. These predictions for a nonadsorbing polyelectrolyte brush have previously been confirmed experimentally. ${ }^{21,51}$ In the present case, dividing the measured brush adsorbed amount by the brush height shows the monomer density in the brush to have been of order $10^{-1} \mathrm{M}$, consistent with the observation that the brush structure altered dramatically when the concentration was raised beyond this point.

The height of a polyelectrolyte brush in the salted brush regime where the electrolyte concentration greatly exceeds the counterion concentration in the brush is given by

$$
h \cong v^{1 / 3} N\left(\frac{a}{s}\right)^{2}
$$

where $N$ is the number of Kuhn statistical steps in the polymer chain, $a$ is the Kuhn step length, $s$ is the interanchor spacing, $v$ is the excluded volume coefficient, which may be thought of as the volume surrounding a single monomer into which other monomers cannot penetrate due to repulsive force, and the symbol $\cong$ means equal to within a numerical prefactor of order unity. In the salted brush regime, $v$ arises from electrostatic repulsion and is given by $v \cong l_{\mathrm{ch}} / \kappa^{2}$, where $l_{\mathrm{ch}}$ is the distance between adjacent charges along the chain and $\kappa$ is the Debye screening length defined by $\kappa^{2}=2 \rho_{0} e^{2} / \varepsilon \varepsilon_{0} k_{\mathrm{B}} T$, where $\rho_{0}$ is the electrolyte number density, $e$ is the elementary charge, $\varepsilon \varepsilon_{0}$ is the electrical permittivity of the solvent, $k_{\mathrm{B}}$ is Boltzmann's constant, and $T$ is the temperature, ${ }^{52}$ so that, using $\Gamma_{\mathrm{b}}=\mathrm{Na}^{3} / \mathrm{s}^{2}$, the brush height obeys ${ }^{50}$

$$
h \propto\left(\frac{\Gamma_{\mathrm{b}}}{\rho_{0}}\right)^{1 / 3}
$$

On changing the electrolyte concentration from $10^{-1}$ to $1 \mathrm{M}$, the known changes in $\rho_{0}$ and $\Gamma_{\mathrm{b}}$, therefore predict a decrease in the brush height by a factor of 0.58 , predicting $h=490$ at $1 \mathrm{M}$, close to the measured value of $540 \AA$, suggesting that the salted brush is indeed a good model for this system. However, on increasing the electrolyte concentration further to $3 \mathrm{M}$ and allowing for the fact that no significant change in $\Gamma_{b}$ was measured, eq 4 predicts $h=340 \AA$, significantly different from the measured value of $530 \AA$. This discrepancy may be attributed to the fact that the excluded $v$ only obeys $v \cong l_{\mathrm{ch}} / \kappa^{2}$ as long as $l_{\mathrm{ch}} / \kappa^{2}>v_{0}$, where $v_{0}$ is the value of the excluded volume coefficient given by $v_{0}=b^{3}(1 / 2-\chi)$, where $b$ is the monomer size and $\chi$ is the Flory-Huggins interaction coefficient. Since the Debye length, $\kappa^{-1}$, is $0.3 \AA$ at $1 \mathrm{M}$, which is less than the monomer size $b, v$ must fall to $v_{0}$ at around this concentration, whereupon it cannot fall any further, accounting for the similarity of the brush heights at 1 and $3 \mathrm{M}$.

Effect of Oppositely Charged Surfactant. This section describes the effect of immersing the poly(METAC) brush in solutions of sodium dodecyl sulfate (SDS), a negatively charged surfactant, of successively increasing concentrations between $10^{-4}$ and $10^{-2} \mathrm{M}$. Prior to these measurements, and after the measurement at $3 \mathrm{M} \mathrm{KNO}_{3}$, the poly(METAC) brush was thoroughly rinsed and the neutron reflectivity profile in pure water $\left(\mathrm{D}_{2} \mathrm{O}\right)$ was measured and showed that the two-region structure associated with $\left[\mathrm{KNO}_{3}\right] \leq 10^{-1} \mathrm{M}$ had been recovered. We can therefore regard the SDS measurements described in this section as having been taken after a "new start" from pure or low electrolyte concentration water.

Figure $7 \mathrm{a}$ (red profile) shows the reflectivity profile from a poly(METAC) brush immersed in $10^{-4} \mathrm{M}$ SDS (nondeuterated) in $\mathrm{D}_{2} \mathrm{O}$, which was readily fitted by a model 


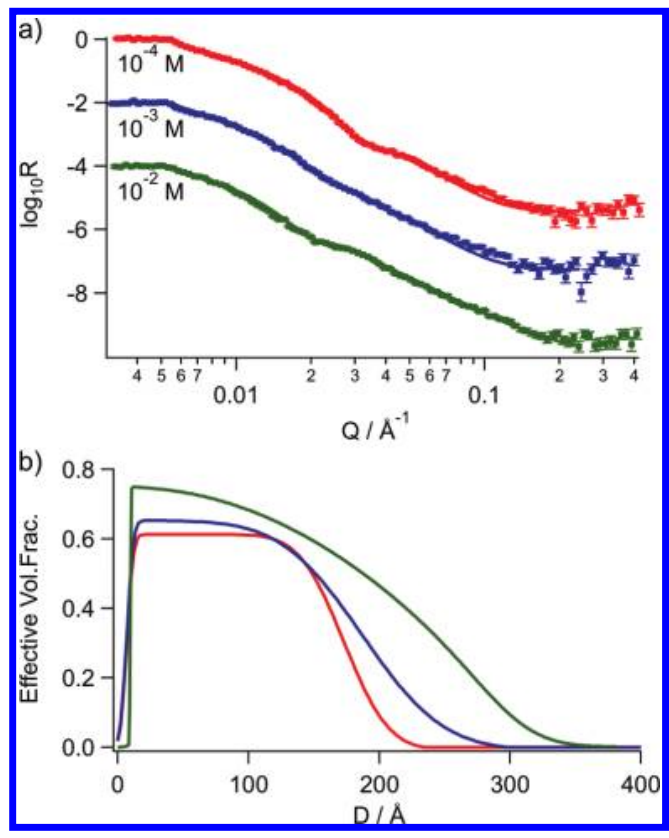

Figure 7. Effect of oppositely charged surfactant on the poly(METAC) layer structure. (a) Neutron reflectivity from poly(METAC) brush in solutions of increasing concentrations of sodium dodecyl sulfate (SDS) in $\mathrm{D}_{2} \mathrm{O}$. Symbols show measured data; solid lines show fits as described in text (for parameter values, see the Supporting Information). Successive profiles are shown displaced vertically for clarity. (b) Lines show effective volume fraction profiles (neglecting differences in scattering length density between SDS and poly(METAC)) derived from solid-line fits in part a with different SDS concentrations represented by the same colors as in part a.

consisting of a single layer with Gaussian roughness; there is no sign of the two-region structure associated with the brush in pure water. The thickness of the layer is only $166 \AA$, indicating that the brush underwent a dramatic collapse with respect to its unperturbed thickness of $830 \AA$. Surfactant was incorporated into the layer: assuming that no poly(METAC) was been removed from the surface, the number of SDS molecules was similar to the number of METAC monomers (Table 1). It

Table 1. Surface Excess of Sodium Dodecyl Sulfate (SDS) per Unit Area of Poly(METAC) Brush, $\Gamma_{S D S}$, as a Function of SDS Concentration, Derived from Solid-Line Fits in Figure 7, and the Corresponding Number Ratio of METAC Monomers to Confined SDS Molecules

$\begin{array}{ccc}{[\mathrm{SDS}] / \mathrm{M}} & \Gamma_{\mathrm{SDS}} / \AA & \text { molecular ratio METAC:SDS } \\ 10^{-4} & 55 & 0.92 \\ 10^{-3} & 71 & 0.72 \\ 10^{-2} & 108 & 0.47\end{array}$

should be noted that the collapse of the brush to less than the blown-dry thickness observed by X-ray reflectometry is not a contradiction, since the blown-dry brush was found to be mostly water. Figure $7 \mathrm{~b}$ (red profile) shows the volume fraction profile of organic material determined by fitting.

Since the collapse of the poly(METAC) brush on addition of SDS occurred at a concentration of only $10^{-4} \mathrm{M}$, whereas electrolyte effects only onset at $\mathrm{KNO}_{3}$ concentrations of order $1 \mathrm{M}$, it is clear that the effect of the SDS cannot have been purely electrostatic. Rather, these results may be attributed to the adsorption of surfactant molecules to the poly(METAC) chains, which neutralizes the poly(METAC) charge, reducing the number of counterions confined within the brush, and also introduces attractive hydrophobic interactions between neighboring dodecyl chains. Similar effects have been reported in weak polyelectrolyte brushes, ${ }^{39,53}$ as well as in a strong spherical polyelectrolyte brush. $^{54}$

As the SDS concentration was increased above $10^{-4} \mathrm{M}$, the amount of SDS confined within the brush increased, as shown in Table 1. At the same time, the effective volume fraction profile began to fall off less rapidly with distance from the surface, as shown in Figure $7 \mathrm{~b}$. The reflectivity profile at $10^{-2} \mathrm{M}$ SDS fell off too gradually to be well-fitted by a single layer with Gaussian roughness and was instead fitted using a quasiparabolic density profile: the model used to fit the profiles measured in pure water but without any appreciable surface region. It can be clearly seen from Figure $7 \mathrm{~b}$ that the overall height of the organic layer increased significantly as SDS concentration was increased from $10^{-4}$ to $10^{-2} \mathrm{M}$, although always remaining well below the unperturbed value of $\sim 1000 \AA$ (Figure $3 b$ ).

This observed increase in brush height with increasing surfactant concentration above $10^{-4} \mathrm{M}$ may be attributed to the likely stiffening of the polymer chains due to the addition of further pendant surfactant molecules, causing the chains to extend further away from the surface. Possible surfactant aggregation within the brush could also have played a role, as the concentrations used are within the range where polymer-surfactant aggregation could occur; the critical micelle concentration of SDS is $8 \times 10^{-3} \mathrm{M}^{55}$ No direct evidence of aggregation was, however, visible in the measured reflectivity profiles, in contrast to a previous study where surfactant multilayers forming parallel to the substrate surface in a weak polyelectrolyte brush gave rise to a Bragg-like peak in the reflectivity profile. ${ }^{39}$ This suggests that any aggregates forming in the poly(METAC)/SDS system are not ordered lamellae.

\section{CONCLUSIONS}

We have synthesized surface-grown polyelectrolyte (poly(METAC)) brushes on a sapphire substrate using a combination of ATRP and silane self-assembled monolayer formation. A kinetic study of dry brush thickness indicates that a saturated and cross-linked self-assembled silane monolayer was produced. Neutron reflectometry measurements of a polyelectrolyte brush at the solid liquid interface showed a dense layer of surface-confined polyelectrolyte, the electrical charge of which caused the remaining polyelectrolyte to stretch away from the surface forming a diffuse, brushlike region with a volume fraction profile in the form of a stretched parabola. When electrostatic interactions were screened out in potassium nitrate solutions of order $1 \mathrm{M}$, some polyelectrolyte was transferred from the brush region to the surface layer, and the brush height decreased. The addition of oppositely charged SDS surfactant at only $10^{-4} \mathrm{M}$ (after rinsing away of electrolyte from the brush) caused the brush to collapse dramatically, destroying the two-region structure. This may be attributed to adsorption of SDS to the oppositely charged polyelectrolyte, which neutralizes the brush charge and introduces hydrophobic attractions between SDS-bearing monomers. Subsequent increases in the surfactant concentration caused the brush height to increase slightly, most likely due to stiffening of the polymer chains by steric interactions between the pendant surfactant molecules. 


\section{ASSOCIATED CONTENT}

\section{S Supporting Information}

Tables listing fitted parameter values from dry X-ray characterization of the poly(METAC) layer and from neutron experiments. This material is available free of charge via the Internet at http://pubs.acs.org.

\section{AUTHOR INFORMATION}

\section{Corresponding Author}

*E-mail: i.dunlop@imperial.ac.uk (I.E.D.); jacob.klein@ weizmann.ac.il (J.K.).

\section{Present Addresses}

${ }^{\ddagger}$ Department of Materials, Imperial College, London, Exhibition Road, London SW11 3HH, U.K.

${ }^{\S}$ School of Physics and Astronomy, University of Edinburgh, Mayfield Road, Edinburgh EH9 3JZ, U.K.

${ }^{\perp}$ Department of Materials, University of Loughborough, Loughborough LE11 3TU, U.K.

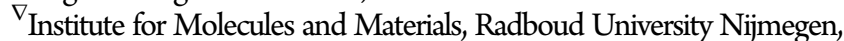
Heyendaalseweg 135, Nijmegen 6525 AJ, The Netherlands.

\section{ACKNOWLEDGMENTS}

We thank Robert Jacobs, John Webster, David Follows, and Robert Cubitt for valuable discussions and assistance and Meng Chen and Frank Schreiber for valuable discussions. We acknowledge funding from EPSRC (IED) and a Royal Society University Research Fellowship (ST).

\section{REFERENCES}

(1) Tuinier, R.; de Kruif, C. G. I. Chem. Phvs. 2002, 117, 1290.

(2) Ligtenberg, M. J. L.; Buijs, F.; Vos, H. L.; Hilkens, J. Cancer Res 1992, 52, 2318

(3) Argueso, P.; Spurr-Michaud, S.; Russo, C. L.; Tisdale, A.; Gipson, I. K. Invest. Ophthalmol. Visual Sci. 2003, 44, 2487.

(4) Carraway, K. L.; Fregien, N.; Carraway, K. L.; Carraway, C. A. C. I. Cell Sci. 1992, 103, 299.

(5) Komatsu, M.; Carraway, C. A. C.; Fregien, N. L.; Carraway, K. L. I. Biol. Chem. 1997, 272, 33245.

(6) Mei, Y.; Sharma, G.; Lu, Y.; Ballauff, M.; Drechsler, M.; Irrgang, T.; Kempe, R. Langmuir 2005, 21, 12229.

(7) Ryan, A. J.; Crook, C. J.; Howse, J. R.; Topham, P.; Geoghegan, M.; Martin, S. J.; Parnell, A. J.; Ruiz-Perez, L.; Jones, R. A. L. J. Macromol. Sci.: Phys. 2005, B44, 1103

(8) Czeslik, C.; Jackler, G.; Hazlett, T.; Gratton, E.; Steitz, R.; Wittemann, A.; Ballauff, M. Phvs. Chem. Chem. Phvs. 2004, 6, 5557.

(9) Padeste, C.; Farquet, P.; Potzner, C.; Solak, H. H. J. Biomater. Sci.. Polvm. Ed. 2006, 17, 1285.

(10) Tran, Y.; Auroy, P.; Lee, L. T. Macromolecules 1999, 32, 8952.

(11) Geoghegan, M.; Ruiz-Perez, L.; Dang, C. C.; Parnell, A. J.; Martin, S. J.; Howse, J. R.; Jones, R. A. L.; Golestanian, R.; Topham, P. D.; Crook, C. J.; Ryan, A. J.; Sivia, D. S.; Webster, J. R. P.; Menelle, A. Soft Matter 2006, 2, 1076

(12) Mir, Y.; Auroy, P.; Auvray, L. Phvs. Rev. Lett. 1995, 75, 2863.

(13) Raviv, U.; Giasson, S.; Kampf, N.; Gohy, J. F.; Jerome, R.; Klein, J. Nature 2003, 425, 163.

(14) Kampf, N.; Gohy, J. F.; Jerome, R.; Klein, J. J. Polym. Sci., Part B: Polvm. Phvs. 2005, 43, 193.

(15) Benz, M.; Chen, N. H.; Israelachvili, J. I. Biomed. Mater. Res. Part A 2004, 71A, 6.

(16) Dunlop, I. E.; Briscoe, W. H.; Titmuss, S.; Jacobs, R. M. J.; Osborne, V. L.; Edmondson, S.; Huck, W. T. S.; Klein, J. I. Phys. Chem. B 2009, 113, 3947.

(17) Muller, F.; Romet-Lemonne, G.; Delsanti, M.; Mays, J. W.; Daillant, J.; Guenoun, P. I. Phvs.: Condens. Matter 2005, 17, S3355.
(18) Biesalski, M.; Johannsmann, D.; Ruhe, J. I. Chem. Phvs. 2004, 120,8807

(19) Balastre, M.; Li, F.; Schorr, P.; Yang, J. C.; Mays, J. W.; Tirrell, M. V. Macromolecules 2002, 35, 9480.

(20) Tran, Y.; Sanjuan, S.; Pantoustier, N.; Perrin, P. Smart Mater. IV (Proc. Vol.) 2007, 6413, G4130.

(21) Sanjuan, S.; Perrin, P.; Pantoustier, N.; Tran, Y. Langmuir 2007, 23,5769 .

(22) Sanjuan, S.; Tran, Y. Macromolecules 2008, 41, 8721.

(23) Fielding, L. A.; Edmondson, S.; Armes, S. P. I. Mater. Chem. 2011, 21, 11773.

(24) Ballauff, M.; Borisov, O. Curr. Opin. Colloid Interface Sci. 2006, $11,316$.

(25) Toomey, R.; Tirrell, M. Annu. Rev. Phys. Chem. 2008, 59, 493.

(26) Claesson, P. M.; Poptoshev, E.; Blomberg, E.; Dedinaite, A. Adv. Colloid Interface Sci. 2005, 114, 173

(27) Ayres, N. Polvm. Chem. 2010, 1, 769.

(28) Matyjaszewski, K. Mol. Crvst. Liq. Crvst. 2004, 415, 23.

(29) Pyun, J.; Kowalewski, T.; Matyjaszewski, K. Macromol. Rapid Commun. 2003, 24, 1043.

(30) Edmondson, S.; Osborne, V. L.; Huck, W. T. S. Chem. Soc. Rev. 2004, 33, 14

(31) Ligoure, C.; Leibler, L. I. Phvs. (Paris) 1990, 51, 1313.

(32) Dunlop, I. E.; Briscoe, W. H.; Titmuss, S.; Sakellariou, G.; Hadjichristidis, N.; Klein, J. Macromol. Chem. Phvs. 2004, 205, 2443.

(33) Titmuss, S.; Briscoe, W. H.; Dunlop, I. E.; Sakellariou, G.; Hadjichristidis, N.; Klein, J. L. Chem. Phvs. 2004, 121, 11408.

(34) Moglianetti, M.; Webster, J. R. P.; Edmondson, S.; Armes, S. P.; Titmuss, S. Langmuir 2010, 26, 12684.

(35) Currie, E. P. K.; Sieval, A. B.; Avena, M.; Zuilhof, H.; Sudholter, E. J. R.; Stuart, M. A. C. Langmuir 1999, 15, 7116.

(36) Tran, Y.; Auroy, P. Eur. Phys. I. E: Soft Matter Biol. Phys. 2001, 5,65 .

(37) Tran, Y.; Auroy, P.; Lee, L. T.; Stamm, M. Phvs.Rev.E 1999, 60, 6984.

(38) Weir, M. P.; Heriot, S. Y.; Martin, S. J.; Parnell, A. J.; Holt, S. A.; Webster, J. R. P.; Jones, R. A. L. Lanomuir 2011, 27, 11000.

(39) Moglianetti, M.; Webster, J. R. P.; Edmondson, S.; Armes, S. P.; Titmuss, S. Langmuir 2011, 27, 4489.

(40) Kobayashi, M.; Yamaguchi, H.; Terayama, Y.; Wang, Z.; Ishihara, K.; Hino, M.; Takahara, A. Macromol. Svmp. 2009, $279,79$.

(41) Kobayashi, M.; Terayama, Y.; Hino, M.; Ishihara, K.; Takahara, A. I. Phys.: Conf. Ser. 2009, 184, 012010.

(42) Thomas, R. K. Annu. Rev. Phvs. Chem. 2004, 55, 391.

(43) Parratt, L. G. Phvs. Rev. 1954, 95, 359.

(44) Bucknall, D. G.; Penfold, J.; Webster, J. R. P.; Zarbakhsh, A.; Richardson, R. M.; Rennie, A.; Higgins, J. S.; Jones, R.; et al. Proceedings of the International Collaboration on Advanced Neutron Sources XIII, PSI Proceedings; Paul Scherrer Institute: Villigen, Switzerland, 1995.

(45) Zhang, X. L.; Taylor, D. J. F.; Thomas, R. K.; Penfold, I. Langmuir 2011, 27, 2601.

(46) Braun, C. Parratt32 or the Reflectometry Tool; Hahn-Meitner Institute: Berlin, 1997.

(47) Quinton, J.; Thomsen, L.; Dastoor, P. Surf. Interface Anal. 1997, 25,931 .

(48) Milner, S. T.; Witten, T. A.; Cates, M. E. Europhvs. Lett. 1988, 5,413 .

(49) Zhulina, E. B.; Borisov, O. V.; van Male, J.; Leermakers, F. A. M. Langmuir 2001, 17, 1277.

(50) Pincus, P. Macromolecules 1991, 24, 2912.

(51) Jia, H. D.; Grillo, I.; Titmuss, S. Lanomuir 2010, 26, 7482.

(52) Barrat, J.-L.; Hansen, J.-P. Basic Concepts for Simple and Complex Fluids; Cambridge University Press: Cambridge, U.K., 2003.

(53) Konradi, R.; Ruhe, J. Macromolecules 2005, 38, 6140.

(54) Samokhina, L.; Schrinner, M.; Ballauff, M. Lanomuir 2007, 23, 3615.

(55) Zhang, X. L.; Penfold, J.; Thomas, R. K.; Tucker, I. M.; Petkov, J. T.; Bent, J.; Cox, A.; Grillo, I. Langmuir 2011, 27, 10514. 